\title{
Liquid Scanning Transmission Electron Microscopy Applied to Study Live Cells
}

\author{
D.B. Peckys, N. de Jonge
}

Molecular Physiology and Biophysics, Vanderbilt University Medical Center, 2215 Garland Ave, Nashville TN 37232-0615

We have developed a methodology to image fully hydrated pristine eukaryotic cells using scanning transmission electron microscopy (STEM), so-called liquid STEM. To be imaged with liquid STEM, the live cells are enclosed in a microfluidic chamber consisting of two silicon microchips with thin windows of silicon nitride. The windows are transparent for electrons and photons, as needed for the recording of both light microscopy and STEM images from the same sample enclosed in the microfluidic chamber. The cells are sealed from the vacuum environment inside the EM, and a continuous flow of buffer can be provided to the cells. Liquid STEM was previously used to image labeled membrane receptors on whole fixed cells in liquid [1].

In the study presented here, we applied liquid STEM to image live eukaryotic cells, i.e. yeast cells (Schizosaccaromyces pombe) and mammalian fibroblasts (COS-7). In both cases we imaged the pristine cells with no other pretreatment than loading with specific live/dead indicator dyes and, for the mammalian cells, an incubation with nanoparticles (NPs), in order to examine the uptake and the intracellular fate of these NPs. In the case of the yeast cells we wanted to test the applicability of liquid STEM imaging for mutant screening. Therefore, we imaged the wild type and different mutants. After their enclosure in the microfluidic chamber and $2-3$ minutes prior to STEM imaging, the cells were tested for viability with light microscopy (see Fig. A, showing FUN-1 red stained, vacuoles in wild type S. pombe cells). The subsequently recorded STEM images revealed intracellular organelles such as the cell wall, the primary and secondary septa, and different types of intracellular vesicles, with a resolution of $30 \mathrm{~nm}$ (Fig. B). Known information of organelle morphology, size, and mass density was used to assign the visible structures in the STEM images to specific yeast organelles. Compared to the STEM images from the wild type, those recorded from the mutants (Fig. C and D) revealed distinct differences in their phenotypes, which would not be visible in such detail with light microscopy. The time frame needed per cell type (yielding STEM images from several cells), was in the order of $1-2 \mathrm{~h}$, this allows for the screening of dozens of mutants in just one week.

Liquid STEM was also used to study NP uptake in mammalian culture cells with the primary aim to assess liquid STEM as a tool for nanotoxicology. NPs have gained substantial attention for medical applications and therefore imply a growing need for examination of their toxic potential on mammalian cells. Ideally, one would study NPs within the context of live cells, but light microscopy does not have sufficient resolution to resolve the NPs. On the other hand, electron microscopy (EM), which is mostly used to record high-resolution images of intracellular NPs, requires a sample preparation that usually cuts the cells into thin sections, a step that highly complicates quantitative studies of NP uptake in cells. For the NP uptake experiments, COS-7 cells (grown on microchips) were incubated with serum protein coated gold-NPs (Au-NPs) of $30 \mathrm{~nm}$ diameter. After $2 \mathrm{~h}$ of incubation, the cells were washed and kept in a $\mathrm{CO}_{2}$ incubator for $24 \mathrm{~h}$. Similar to the yeast cells, the cells were loaded with a specific live/dead indicator dye (calcein AM), enclosed in a microfluidic chamber and their viability was tested with fluorescence microscopy. In a series of previous experiments, we had tested the cells for survival in the microfluidic chamber and did not find any signs of cellular damage or cell death after enclosure times up to $2.5 \mathrm{~h}$. An example 
is shown in Fig. E and F, depicting COS-7 cells with similar intensity of green fluorescence before and 55 min after enclosure. Cells that had taken up NPs where enclosed in the microfluidic chamber, checked for viability and also imaged with bright field light microscopy. Distinct intracellular dark spots were evident (Fig. G). Subsequent STEM imaging revealed that these spots consisted of large intracellular clusters; made from round structures that were filled with Au-NPs (Fig. H). A cluster contained $>150$ of such NP-filled structures. Due to their approximate round shapes, their filling with Au-NPs and their size distribution we suggest that the NP-filled structures are lysosomes, organelles reported to be involved in the storage of other types of NPs as well [2]. We here demonstrate the applicability of liquid STEM to study intracellular organelles in whole cells in their living state. The recorded STEM images have a resolution that lies about a factor of ten above the light microscopic resolution. In case of the yeast cells, the contrast was achieved on unlabeled, pristine cells; in the fibroblast cells, we imaged NPs probably contained in lysosomes. Although all cells were dead after a STEM image was taken, we could not detect signs of radiation damage in the first STEM image that was taken of a cell. We therefore assume that the STEM images reflect the situation in the living cell, at least in the range of resolution we achieved. [3]

\section{References}

[1] N. de Jonge et al., Proc. Natl. Acad. Sci. 106 (2009) 2159.

[2] L. W. Zhang et al., Toxicol Sci 110 (2009) 138.

[3] We are grateful to T.E. McKnight, P. Mazur, and Protochips Inc., NC. This research was in part conducted at the SHaRE User Facility, which is sponsored by the Division of Scientific User Facilities, Office of Basic Energy Sciences, U.S. Department of Energy. Research supported by Vanderbilt University Medical Center, by NIH grants R01RR018470 (to P. Mazur), and 1R43EB008589 (to S. Mick).

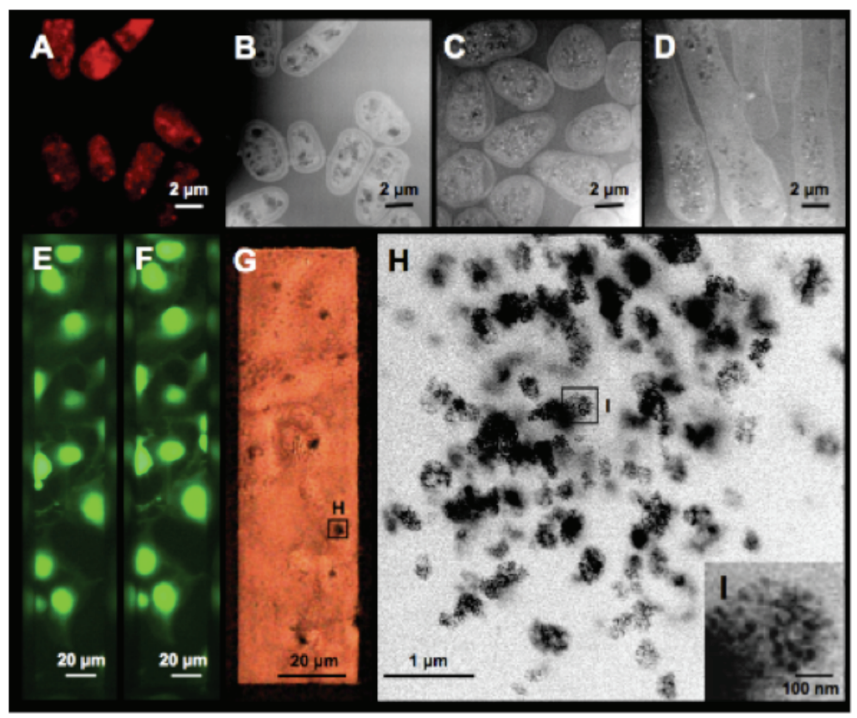

FIG. 1. Light and electron microscopic images from live cells in a microfluidic chamber. (A-D) S. pombe yeast cells, (E-H) COS-7 fibroblast cells. (A) Fluorescence image of living yeast cells. (B) Same cells imaged in the STEM. (C, D) STEM images of two mutants of S. pombe cells. (E) Fluorescence image of living COS-7 cells before enclosure in the microfluidic chamber. (F) 55 min after enclosure the cells still exhibited the same amount of fluorescence. (G) Bright field light microscopic image of COS-7 cells that had accumulated nanoparticles (Au-NPs). Intracellular dark spots are standing out. A STEM image was taken from the spot marked with a square. (H) The STEM image of the marked spot revealed that it consisted of a large cluster of round structures filled with Au-NPs. One of these structures is marked with a square and shown in detail in (I). 